\title{
Short Communication: Using RAPD technique to evaluate genetic diversity of longan (Dimocarpus longan) population in Vietnam
}

\author{
VIET THE HO ${ }^{1, \vartheta}$, QUOC NGUYEN NGO ${ }^{2}$ \\ ${ }^{1}$ Faculty of Biotechnology and Environmental Technology, Ho Chi Minh City University of Food Industry. 140 Le Trong Tan Street, Tan Phu District, \\ Ho Chi Minh City, Vietnam, Tel.: +84-933-025-523, "email: thehv@entp.edu.vn \\ ${ }^{2}$ Ho Chi Minh City University of Science, 227 Nguyen Van Cu, District 5, Ho Chi Minh City, Vietnam
}

Manuscript received: 27 September 2017. Revision accepted: 20 October 2017.

\begin{abstract}
Ho VT, Ngo QNg. 2017. Short Communication: Using RAPD technique to evaluate genetic diversity of longan (Dimocarpus longan) population in Vietnam. Biodiversitas 18: xxxx. Longan (Dimocarpus longan L.) is an important fruit plant in Vietnam where several cultivars and landraces are being cultivated. Presently, the identification of longan cultivars and landraces is mainly based on personal experience relying on morphological traits leading the difficulty of genetic conservation and longan breeding. In this study, genetic richness and relativeness of 36 longan accessions collected from different provinces in Vietnam were evaluated by using 30 Random Amplified Polymorphic DNA (RAPD) primers. Our results reveal that there is the large variation of genetic background of studied longan accessions with separate groups in the obtained dendrogram. We also identified the combination of different RAPD markers could help to identify 15 longan genotypes. The results from this project could provide valuable information which is necessary for classifying, identifying plant origins, breeding and conserving programs of longan in Vietnam.
\end{abstract}

Keywords: Dimocarpus longan, genetic diversity, molecular markers, RAPD

\section{INTRODUCTION}

Longan (Dimocarpus longan L.) is an important fruit tree in Vietnam with a total area of 75,200 ha and the annual production is around 620,000 tons. Despite its importance, breeding and conserving activities of the plant in this country is limited and the origin of this plant in the country is uncertain (FAO 2004). Longan varieties in this area are mostly identified by their local names mostly based on where plants are grown or notable plant features without clear clue about their origin or genetic characteristics. Vernacular names of longan varieties are just based on plant or fruit features which are not accurate because these characteristics can change with environment and culture (Qiu and Zhang 1996). Additionally, studies on longan is mainly depended on agronomical and morphological characteristics (FAO 2004; Hong and Chau 2004; Hoang 2011). Thus, historical identification of plant is challenged because morphological observations are highly dependent on environmental conditions and developmental stages of a plant (Yonemoto et al. 2006).

Presently, RAPD was shown as highly potential technique to genetic study of plant without adequate prior genetic information such as longan. In 2001, by using RAPD Chen and Liu (2001) re-identified new longan cultivar "Reying 17" which was previously considered belong to "Shixia" cultivar. Also in this study, they also detected several cultivar names are replicated because of the identical genetic composition such as "Dazao" and Zaohong", "Feiziziao" and "Zimai", or "Baiye" and "Guahong" in 62 lichi and longan accessions studied. More recently, Yang and colleagues (2013) were able to generate specific loci which are later used to distinguish cultivated longan $D$. longan from its wild and poisonous relative $D$. confinis. Moreover, Mei's research group used successfully this technique to distinguish different longan accessions from geographical origins in China (Mei et al. 2014). The obtained results in this study will be useful for genetic conservation and breeding purposes. The accuracy of genotype identification will be crucial help for farmers to set up new longan orchards because they can significantly reduce the maintaining of undesirable plants. Furthermore, the finding markers tightly linking to favorable cultivars among accessions will also pave the way for breeding programs to produce new longan varieties with better yield and higher fruit quality of longan in Vietnam.

\section{MATERIALS AND METHODS}

\section{Sample collection and DNA extraction}

Total of 36 leaf samples was collected from North to South of Vietnam (Figure 1 and Table 1). Leaf samples were dried and stored in silica gel until use. DNA was extracted with CTAB method (Cetyl Trimethyl Ammonium Bromide) as Madhou et al. (2013). PCR reactions were 


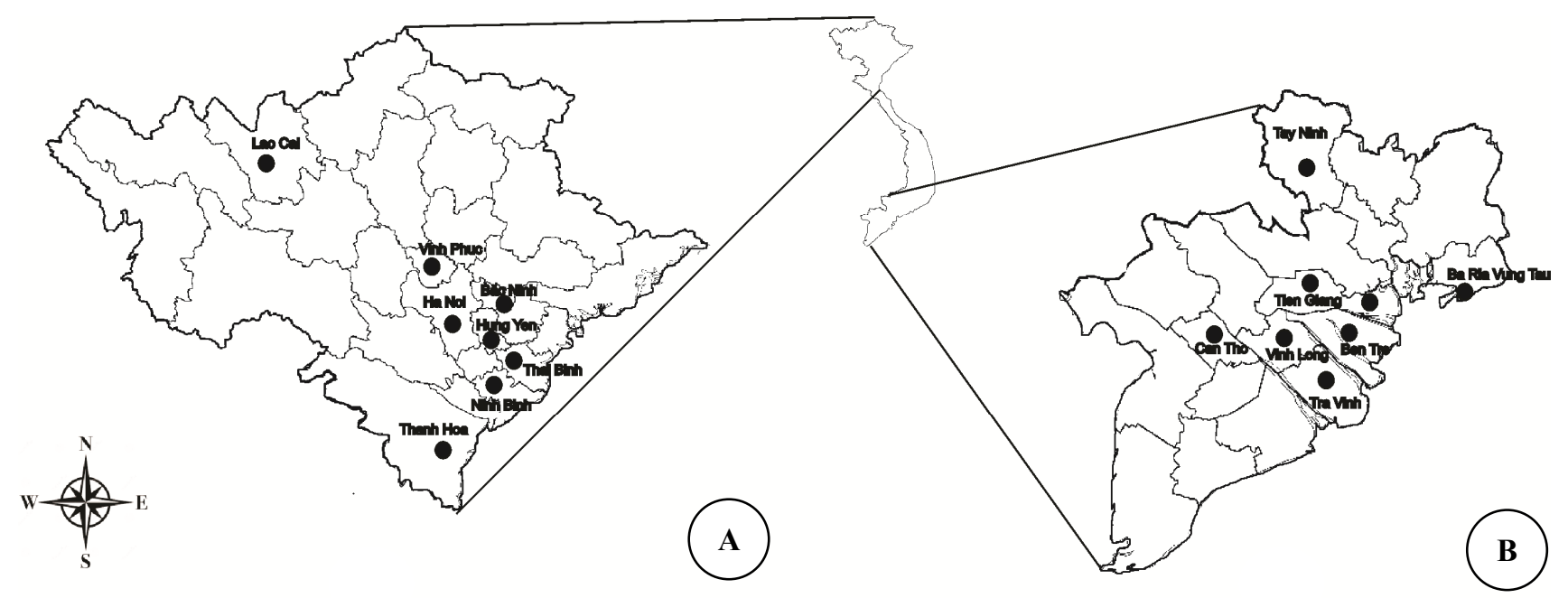

Figure 1. Targeted areas for sample collection of Dimocarpus longan in Vietnam. (A: Northern region; B: Southern region; black dots indicate the sample collection sites in this study)

Table 1. Samples collected for genetic characterization in present study

\begin{tabular}{cll}
\hline Code & Name of cultivar/variety/local name & Collected location \\
\hline 1 & Nhan To Chua Hien & Hung Yen \\
2 & Nhan Long Chin Som & Plant Genetic \\
& & Resources of Vietnam \\
3 & Nhan Xuong Com Vang & Tay Ninh \\
4 & Nhan Chin Muon & Hung Yen \\
5 & Nhan Chin Muon & Ha Noi Agriculture \\
& & University \\
6 & Nhan Thuan Thanh Bac Ninh & Bac Ninh \\
7 & Nhan Co Bac Ninh & Bac Ninh \\
8 & Nhan Gia Vien Ninh Binh & Ninh Binh \\
9 & Nhan dai Quang Xuong & Thanh Hoa \\
10 & Nhan dai TP Ninh Binh & Ninh Binh \\
11 & Nhan Duong Phen & Fruit and Vegetable \\
& & Research Insitute \\
12 & Nhan Xuong Com Vang & Vung Tau \\
13 & Nhan Cui Thai Binh & Thai Binh \\
14 & Nhan dai Lao Cai & Lao Cai \\
15 & Nhan Duong Phen Chua Hien & Hung Yen \\
16 & Nhan Thai Tay Ninh & Tay Ninh \\
17 & Nhan Tieu Hue Tay Ninh & Tay Ninh \\
18 & Nhan Ha Tay & Fruit and Vegetable \\
& & Research Insitute \\
19 & Nhan Xuong Com Vang Ben Tre & Ben Tre \\
20 & Nhan Xuong Com Vang Vinh Long & Vinh Long \\
21 & Nhan Idor Can Tho & Can Tho \\
22 & Nhan My Ben Tre & Ben Tre \\
23 & Nhan Huong Chi & Ha Noi University of \\
& & Agriculture \\
24 & Nhan Da Bo Long Huong & Ba Ria \\
25 & Nhan dai TP Ninh Binh & Vinh Phuc \\
26 & Nhan Long Chin Som Thanh Hoa & Thanh Hoa \\
27 & Nhan dai Cau Lim Ninh Binh & Ninh Binh \\
28 & Nhan Mien Thiet & Fruit and Vegetable \\
& & Research Insitute \\
29 & Nhan Huong Chi & Fruit and Vegetable \\
& & Research Insitute (Ha \\
30 & Nhan Xuong Com Vang & Noi) \\
& & Southern Fruit \\
31 & Nhan dai Quoc Tu Giam & Research Institute \\
32 & Nhan dai Ben Tre & Ha Noi \\
33 & Nhan tieu da bo & Long An \\
34 & Thanh Nhan Ben Tre & Tra Vinh \\
35 & Nhan dai Ba Dinh Ha Noi & Ben Tre \\
36 & Nhan dai But Thap & Ha Noi \\
& & Bac Ninh \\
\hline
\end{tabular}

performed in a total volume of $20 \mu \mathrm{L}$ containing $30 \mathrm{ng}$ DNA, $1 \mathrm{X}$ reaction buffer, $2 \mathrm{mM} \mathrm{MgCl}, 0.3 \mu \mathrm{M}$ of each primer, $200 \mu \mathrm{M}$ of each dNTP, 1 unit of Taq polymerase and sterile water to the final volume. PCR amplification was carried out as follows: initial denaturation at $95^{\circ} \mathrm{C}$ for 2 minutes; after that followed by 40 cycles of $30 \mathrm{~s}$ at $95^{\circ} \mathrm{C}$, $30 \mathrm{~s}$ at $35^{\circ} \mathrm{C}, 54 \mathrm{~s}$ at $72^{\circ} \mathrm{C}$ and final extension for $5 \mathrm{~min}$ at $72^{\circ} \mathrm{C}$. PCR amplification was then separated by electrophoresis in $1.5 \%$ agarose gel in $1 \mathrm{X}$ TAE buffer, and stained with $0.5 \mu \mathrm{g} / \mathrm{mL}$ Gelred TM loading buffer then visualized under ultraviolet light. The primers for PCR reactions were chosen as described by Yonemoto et al. (2006) and Mei et al. (2014) shown in Table 2.

\section{RAPD reactions and data analysis}

After electrophoresis, only clear and reproducible bands with size from 200 to 2,500 bp among two repeated PCR were considered for data analysis (Figure 2). The quality of RAPD markers is evaluated through polymorphism information content (PIC) value described by Chesnokov and Artemyeva (2015) as the formula:

$$
\mathrm{PIC}_{\mathrm{j}}=1-\sum_{i=1}^{n} P i^{2} .
$$

Where $i$ is $i$-th allele of the $j$-th marker, $n$ is the number of the $j$-th marker's alleles, $P$ is allele frequency.

PCR products were scored as "1" for the presence and " 0 " for absence in specific position. The dendrogram was built based on the unweighted pair group method with arithmetic mean and the algorithm (UPGMA) was produced by using SAHN module in NTSYSpc 2.1 package (Rohlf 2000). Principal Coordinate Analysis (PCoA) was performed based on RAPD data to have better understanding about similarity among accessions by using PCoA package in NTSYS-pc 2.1 (Ibrahim et al. 2017). 
Table 2. List of RAPD primers to analyze genetic diversity of longan in Vietnam

\begin{tabular}{ll}
\hline Primer & Primer sequence \\
\hline SBS-A15 & TTCCGAACCC \\
SBS-Q19 & CCCCCTATCA \\
SBS-M1 & GTTGGTGGCT \\
SBS-Q12 & AGTAGGGCAC \\
A02 & GCCAGCTGTACG \\
A03 & TGCCTCGCACCA \\
A13 & CTCAGCGATACG \\
A18 & GACCTGCGATCT \\
A19 & AAGGCGCGAACG \\
A37 & AGCGCGGCAAAA \\
A39a & CCTGAGGTAGCT \\
A58 & GTCATGCCTGGA \\
A62 & TCGTCCGGAGAT \\
C11 & AGGTACGCCCGA \\
C16 & CGCCCTGCAGTA \\
C31 & GAGTTGCCCGGA \\
C34 & TACCCAGGAGCG \\
C59a & CGCGTTCGTGGA \\
C62a & CCATCCGCACGA \\
C82 & ATCGTCACCCCG \\
D12 & CTGGTCTCTGGG \\
D29 & GACCCGGAACGA \\
D38 & AAGCTCGACGGG \\
D41 & GAGACCCGTCGA \\
D50 & GACTCGCGGTCT \\
D53 & GCCGCGGAACTA \\
D84 & AGACACACGGGC \\
F10a & CAGGCCGAAGTC \\
F44a & GGTGTCTTGCGG \\
Z1 & CCGGTGCCTTCT \\
\hline
\end{tabular}

Table 3. Test results of RAPD primers used in present study

\begin{tabular}{llll}
\hline Primer & Band number & Polymorphism (\%) & PIC value \\
\hline SBS-A15 & 12 & 58.3 & 0.43 \\
SBS-Q19 & 11 & 27.3 & 0.27 \\
SBS-M1 & 9 & 55.6 & 0.51 \\
SBS-Q12 & 9 & 44.4 & 0.47 \\
A02 & 10 & 30.0 & 0.42 \\
A03 & 8 & 50.0 & 0.57 \\
A13 & 11 & 45.5 & 0.43 \\
A18 & 8 & 25.0 & 0.41 \\
A19 & 10 & 70.0 & 0.58 \\
A37 & 11 & 18.2 & 0.47 \\
A39a & 9 & 44.4 & 0.37 \\
A58 & 13 & 38.5 & 0.56 \\
A62 & 12 & 66.7 & 0.75 \\
C11 & 10 & 40.0 & 0.51 \\
C16 & 14 & 21.4 & 0.69 \\
C31 & 4 & 25.0 & 0.39 \\
C34 & 6 & 33.3 & 0.63 \\
C59a & 8 & 37.5 & 0.40 \\
C62a & 7 & 42.9 & 0.45 \\
C82 & 12 & 41.7 & 0.40 \\
D12 & 10 & 70.0 & 0.39 \\
D29 & 7 & 57.1 & 0.72 \\
D38 & 8 & 37.5 & 0.68 \\
D41 & 10 & 20.0 & 0.36 \\
D50 & 12 & 16.7 & 0.34 \\
D53 & 7 & 57.1 & 0.43 \\
D84 & 9 & 77.8 & 0.33 \\
F10a & 9 & 66.7 & 0.39 \\
F44a & 6 & 50.0 & 0.52 \\
Z1 & 12 & 41.7 & 0.35 \\
\hline & & &
\end{tabular}

\section{RESULTS AND DISCUSSION}

Total of 36 longan accessions was characterized with 30 RAPD primers. We found that all primers used in this study generated high polymorphism among different accessions (Table 3) with clear amplification and high reproducibility after two replications. Tested primer generated from 4 to 14 amplifications ranging from 200 to $1500 \mathrm{bp}$ with a large variation of total and polymorphic bands from 18.2 to $77.8 \%$. All primers show PIC value from 0.27 to 0.75 (Table 3), meaning that all of these used primers are suitable for genetic characterization of longan collected in research areas. As PIC classification of Botstein et al. (1980) as following: highly informative if $\mathrm{PIC}>0.5$; reasonably informative if $0.5>\mathrm{PIC}>0.25$ and slightly informative if $\mathrm{PIC}<0.25$.

The combination of different amplicons from this study shows the high discrimination capacity of RAPD markers, relying on RAPD data, we could successfully identify several important longan cultivars by using specific amplification from either single or combined different primers. For example, by using primer D38, there is a candidate allele at $600 \mathrm{bp}$ which could be used to identify Nhan Xuong Com Vang Tay Ninh province as shown in line 3 (Figure 2). This is a high-value cultivar because of its superior feature as high resistance to diseases and high fruit quality. Additional bands with size of $1000 \mathrm{bp}$ and $1100 \mathrm{bp}$ could also be potential for recognizing two longan landraces from Thuan Thanh district, Bac Ninh provine (line 6) and Gia Vien district, Ninh Binh province (line 8), respectively. The total RAPD amplification can be utilized to different 15 longan genotypes are presented in Table 4. The ability of RAPD method to differentiate longan genotypes early reported in the previous study of Yenomoto and colleagues (Yenomoto et al. 2006) because RAPD is able to produce a higher number of genetic loci than morphological and biochemical markers. Moreover, RAPD markers were also successfully applied in testing the fidelity of micropropagation of different plants (Rani et al. 1995). Thus, the specific bands obtained in our study could be used to identify the origin of materials for longan breeding programs at least in studied areas. In spite of numerous genetic information was generated, the RAPD makers used in this study is not able to distinguish completely all longan accessions. We suggest that the estimation of genetic diversity by RAPD marker is highly influenced by specific genome of selected accession and by the specific RAPD primer examined. In order to get more accurate result from RAPD markers, future studies need use higher number of marker to cover higher density on genome of plant to measure the genetic variation more exactly. Another reason should be noted that the most samples in this study are from cross-pollinated plants, consequently, the obtained genetic variability is highly depended on individual plants.

Based on obtained RAPD data, the relatedness of 36 longan genotypes was analyzed with NTSYSpc 2.1 amplification profiles were compared to generate a similarity matrix and showed in Table 5. Overall, the genetic similarity varied from 0.46 to 0.95 . The lowest 
similarity value (0.46) was observed between Nhan Dai Ben Tre (32) and Nhan Dai Thanh Pho Ninh Binh (10) and the highest similarity value $(0.95)$ was observed between Nhan Xuong Com Vang Tay Ninh (3) and Nhan Xuong Com Vang Ben Tre (19). This result is reasonable because Nhan Dai Ben Tre (32) and Nhan Dai Thanh Pho Ninh Binh (10) were collected from South and North regions, respectively. Whereas the close relationship between Nhan Xuong Com Vang Tay Ninh (3) and Nhan Xuong Com Vang Ben Tre (19) is not surprised because both of them are from the southern region and belong to Nhan Xuong cultivar suggesting these two accessions have a common ancestor.

The dendrogram was then built based on similarity matrix which showed clear three main groups. The first group consists of 16 genotypes, the second group consists of 16 genotypes and the last group includes 4 genotypes. We found that there is the difference of genetic composition of longans in the North and the South of Vietnam. It is interesting that all group I consists only accessions collected from North of Vietnam. Group III includes only longan from the Southern area with four accessions namely Nhan Dai Ben Tre (32), Nhan Tieu Da Bo Tra Vinh (33), Thanh Nhan Ben Tre (34) and Thanh Nhan Bac Lieu (35), whereas there is the mixture among accessions collected from both areas. This could explain that longan breeders might have used materials from same desirable plants and grafted to several different plants or multiplied and sell to different places. Interestingly, we also found that some accessions from the North and the South showing high similarities such as Nhan My Ben Tre (22) and Nhan Bac Ninh (7). This could be the exchange longan seedlings from different places because in Vietnam farmer is relatively willing to accept new cultivars from different places to grow in their gardens because this will increase income due to the new product in market. It is also common when in the market or seedling nursery, there are several longan accessions with origin from foreign countries is sold.

Our results are in agreement with the previous publication of Yonemoto et al. (2006) where they found the high genetic diversity of longan population in Thailand. Interestingly, among 22 longan genotypes, their study also included four longan accessions from Vietnam consisting of two well-known accessions: Xuong Com Vang and Tieu Da Bo, together with two unknown identified accessions namely Ishigaki-2 and Ishigaki- 2. Using RAPD marker and built dendrogram with NTSYSpc 2.2, they also reported that two unknown identity longan accessions from Vietnam belong to separate group and have far genetic distance with other accessions from Vietnam, Thailand, China, United State, Taiwan, Malaysia, and Japan. On the other hand, Xuong Com Vang and Tieu Da Bo cultivars were also grouped together and processing genetic similarity with their nearest neighbor just as low as 0.824 .

The relationship among different longan accession was also evaluated by PCoA, the first two coordinates were shown in Figure 4. On the basis of the first coordinate, up to $83 \%$ was explained by the first two axes accounting for 50 and $33 \%$ of dimension 1 and dimension 2, respectively.

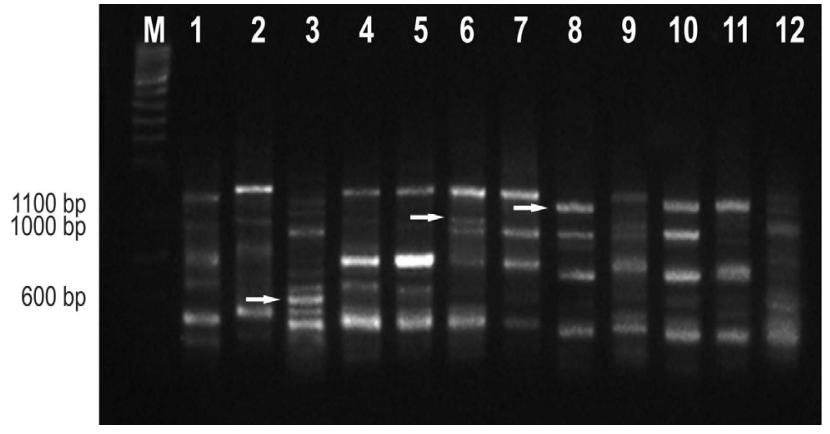

Figure 2. Representative RAPD result with D38 primer. (The arrow shows the candidate amplification marker to distinguish specific longan cultivars with other longan accessions; the number is corresponding to sample code in Table 1; M: DNA ladder)

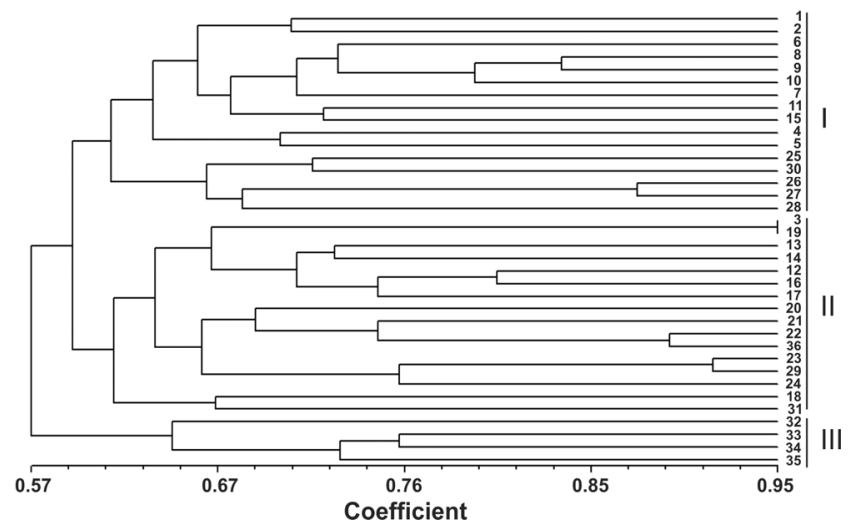

Figure 3. Dendrogram generated by using 30 RAPD markers to show the genetic relativeness of 36 longan accessions. This dendrogram was developed using UPGMA cluster procedure of NTSYSpc 2.2. The scale shown at the bottom is the measure of genetic similarity. The scale shown at the bottom is the measure of genetic similarity. (The number is corresponding to sample code in Table 1).

Table 4. Longan accessions-specific amplified bands produced by selected RAPD primers

\begin{tabular}{lll}
\hline Accession name, collected place & Primer & $\begin{array}{l}\text { Size of candidate } \\
\text { amplification (bp) }\end{array}$ \\
\hline Nhan Duong Phen, Hung Yen & D29 & 900 \\
Nhan Mien Thiet, Khoai Chau & C59a & 1000 \\
Nhan My, Ben Tre & C82 & 2500 \\
Nhan Thai, Tay Ninh & A58 & 1200 \\
& C16 & 500 \\
Nhan Tieu Hue & A58 & 1200 \\
& D29 & 400 \\
Nhan Xuong Com Vang, Tay Ninh & D16 & $800 ; 1000$ \\
& C31 & 600 \\
Nhan Xuong Com Vang, Vinh Long & C82 & 300 \\
Nhan Xuong Com Vang, SOFRI & C34 & $500 ; 2000$ \\
& D38 & 400 \\
Nhan To Chua Hien, Hung Yen & A62 & 900 \\
Nhan Long Chin Som, Hung Yen & A62 & 1200 \\
Nhan Ha Tay & D29 & 300 \\
Nhan Huong Chi, Hung Yen & C34 & 500 \\
Nhan Ben Tre & D38 & 1200 \\
Nhan Thuan Thanh, Bac Ninh & D38 & 1000 \\
Nhan Gia Vien, Ninh Binh & D38 & 1100 \\
\hline
\end{tabular}


Table 5. Simple matching coefficients of similarity among 34 genotypes of longan (Dimocarpus longan)

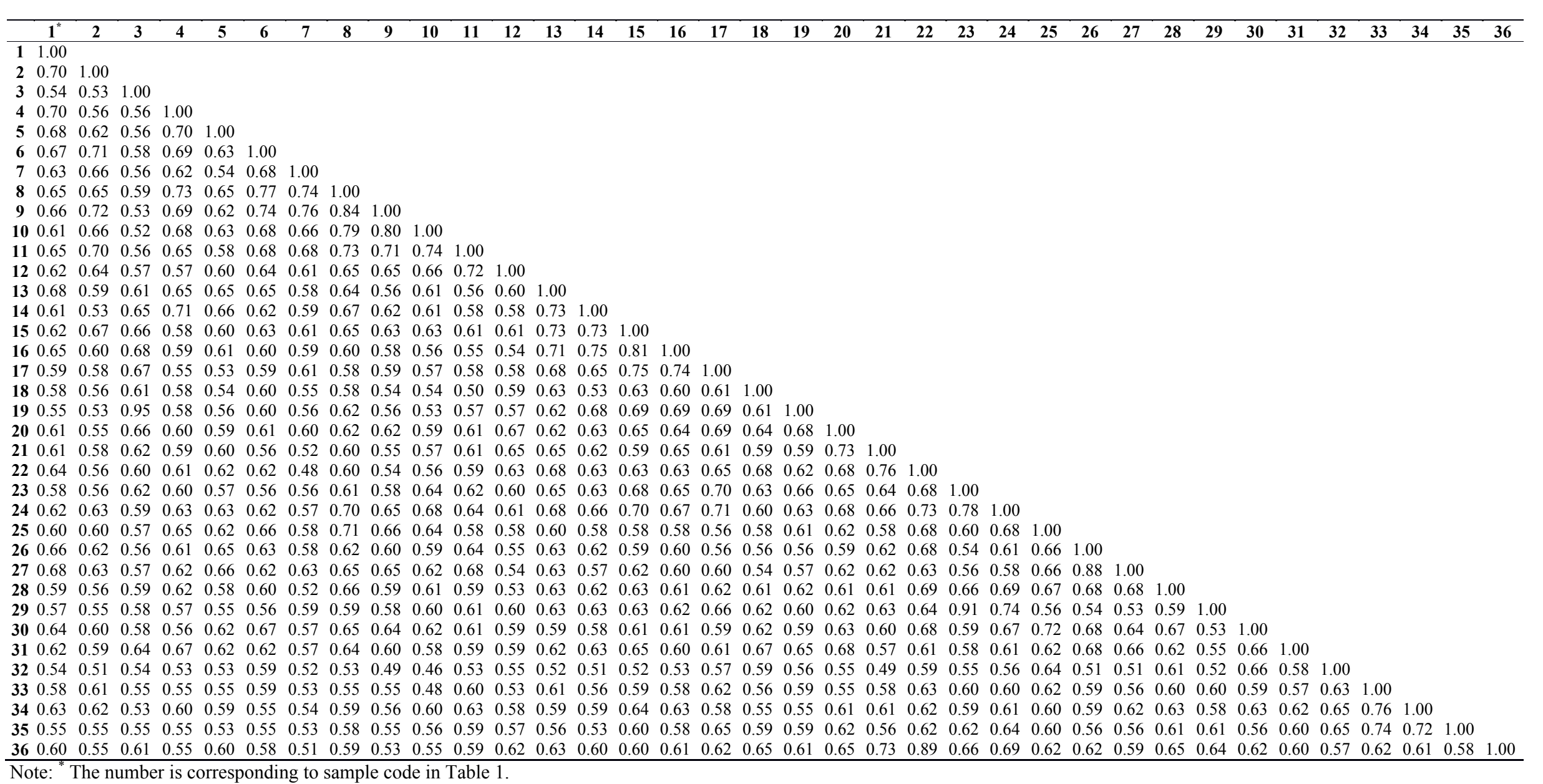




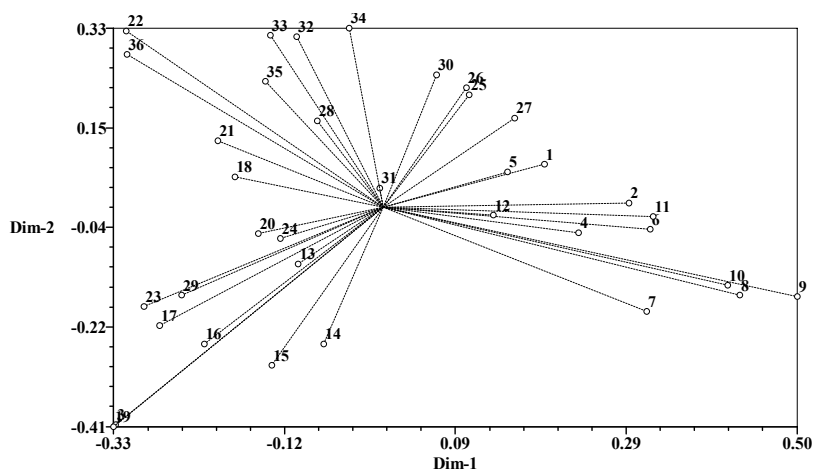

Figure 4. Two-dimensional plot of principle coordinates analysis depicting the genetic relatedness of thirty-six longan genotypes (The number is corresponding to sample code in Table 1)

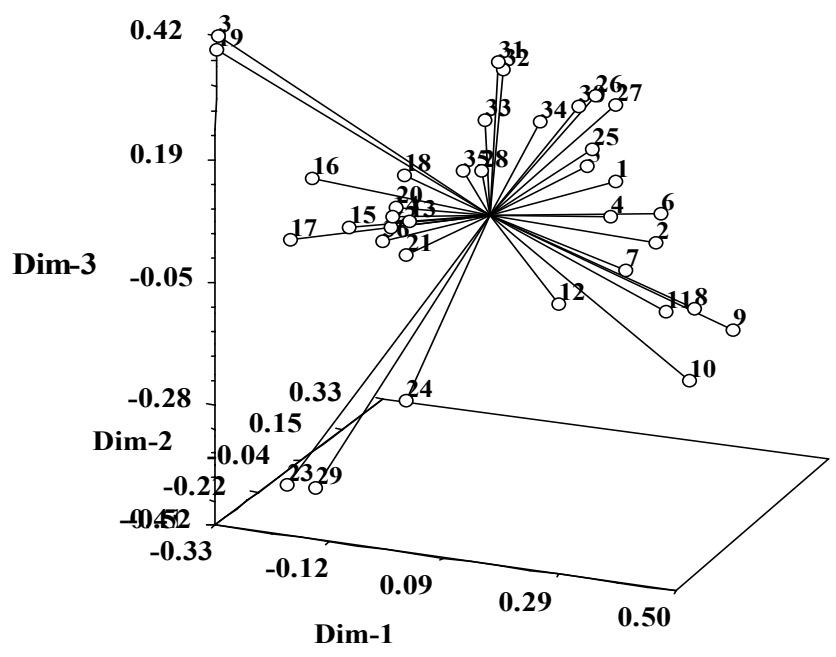

Figure 5. Principle coordinate plot for the first three principal coordinates estimated for RAPD markers of the thirty-six longan genotypes (The number is corresponding to sample code in Table 1)

The result of this analysis is relatively corresponding with dendrogram analyzed by UMPMA (Figure 3). Among total accessions, Nhan Xuong Com Vang Tay Ninh (3) and Nhan Xuong Com Vang Ben Tre (19) were grouped at distant position of dendrogram and which is also repeated after PCoA analysis at 2 dimensional and 3-dimensional plot as shown in Figure 4 and Figure 5, respectively. Thus, PCoA can be used for further confirmation of genetic diversity by using UPGMA method as described previously by Johar et al. (2017).

RAPD is considered as a frontline technique to study organism with limited prior genetic knowledge such as longan. In this project, we identified the usefulness of this technique in investigating genetic diversity of longan in Vietnam. By using 30 RAPD primers to analyze 36 longan samples, the collected samples were divided into 3 main groups showing the close relativeness of accessions in different places. We also found several specific RAPD bands which could be further analyzed to use as specific markers to identify 15 specific longan varieties. The obtained results in this study will be important information which will be useful for plant breeders to use for several purposes such as classification, conservation and longan breeding programs in Vietnam.

\section{ACKNOWLEDGEMENTS}

Authors are thankful to Southeast Asian Regional Center for Graduate Study and Research in Agriculture (SEARCA) for financial support under Seed Fund for Research and Training programs. We also thank Faculty of Biotechnology and Environment Engineering, Ho Chi Minh City University of Food Industry for providing research facilities.

\section{REFERENCES}

Botstein D, White RL, Skolnick M, Davis RW. 1980. Construction of a genetic linkage map in man using restriction fragment length polymorphism. Am J Hum Genet 32: 314-331.

Chen YZ, Liu CM. 2001. Identification and analysis of 5 longan varieties by RAPD. China Fruits 4: 28-29.

Chesnokov YV, Artemyeva AM. 2015. Evaluation of the measure of polymorphism information of genetic diversity. Agric Biol 50 (5): 571-578.

FAO. 2004. Fruits of Vietnam. Corporate document repository. Food and agriculture organization of the united nations, Regional Office for Asia and the Pacific, Bangkok, Thailand.

Hoang TVH. 2011. Development of fruit-tree in Dong Thap province: Practices and Solutions. Scientific journal of Ho Chi Minh City University of Pedagogy 26: 74-79. [Vietnamese]

Hong BM, Chau NM. 2004. Longan production in Vietnam. In: Chomchalow N, Sukhavibul N (eds.) Proc. 2nd Int. Symp. Lychee, Longan, Rambutan and Other Sapindaceae Plants. Acta Horticulturae.

Ibrahim KS, Gurusubramanian G, Zothansanga, Yadav RP, Senthi Kumar N, Pandian SK, Borah P, Mohan S. 2017. Bioinformatics- A student's Companion. Springer Science + Bussiness Media, Singapore.

Johar V, Kajla S, Dhillon RS, Bhatia P. 2007. Evaluation of genetic diversity using random amplification polymorphic DNA (RAPD) markers in Melia dubia Cav. Indian J Biotechnol 16:76-83.

Madhou M, Normand F, Bahorun R, Hormaza JI. 2013. Fingerprinting and analysis of genetic diversity of litchi (Litchi chinensis Sonn.) accessions from different germplasm collections using microsatellite markers. Tree Genet Genomes 9: 387-396.

Mei ZQ, Fu SY, Yu HQ, Yang LQ, Duan CG, Liu XY, Gong S, Fu JJ. 2014. Genetic characterization and authentication of Dimocarpus longan Lour. Using an improved RAPD technique. Genet Mol Res 13 (1): 1447-1455.

Qiu WL, Zhang HZ. 1996. Encyclopedia of China Fruits: Longan. China Forestry Press, Beijing, China.

Rani V, Parida A, Raina SN. 1995. Random amplified polymorphic DNA (RAPD) markers for genetic analysis in microprogated plants of Populus deltoires Marsh. Plant Cell Rep 14: 459-462.

Rohlf FJ. 2000. NTSYS-pc: Numerical taxonomy and multivariate analysis system, version 2.2. Exeter Software, Setauket, New York.

Yang L, Fu S, Khan MA, Zeng W, Fu J. 2013. Molecular cloning and development of RAPD-SCAR markers for Dimocarpus longan variety authentication. Springerplus 2:5 01. DOI: 10.1186/2193-1801-2-501

Yonemoto Y, Chowdury AK, Kato H, Macha MM. 2006. Cultivar identification and their genetic relationships in Dimocarpus longan subspecies based on RAPD markers. Sci Hort 109:147-152. 